\title{
Micro-Doppler modeling and simulation research of several typical micro motion forms
}

\author{
Haiyang Zhang, Shen $\mathrm{Li}^{\mathrm{a}}$ and Zheng Zheng \\ School of Opto-Electronics, Beijing Institute of Technology, Beijing 100081, China
}

\begin{abstract}
A Micro-Doppler mathematical model of the point target and radar is established in the four kinds of micro motion forms: vibration, rotation, tumbling and cone. A new method of detecting Micro-Doppler features is introduced: double pulse laser coherent detection, combining algorithm principle and mathematical model, the echo signals of four kinds of micro motion targets are simulated separately to obtain the Micro-Doppler time frequency spectrum of the target, during simulation, input parameters of each target are changed to analyze its influence on Micro-Doppler frequency. Then by comparing the difference of time frequency spectrum of different targets, the Micro-Doppler features are studied, which are important references to distinguish the different micro motion forms. This research project provides some references for the micro motion target detection, feature extraction and target identification.
\end{abstract}

Keywords: micro motion target; double pulse laser coherent detection; Micro-Doppler time frequency spectrum; Micro-Doppler feature.

\section{Introduction}

After radar Micro-Doppler phenomenon was found, the micro motion feature has been widely concerned in the detection and recognition of radar targets. Micro motion can be defined as a small non uniform motion or motion component about target or some part of the target in the radical direction relative to the radar. Micro motion can be easily found in nature, such as the swing of human hands and feet; the vibration of a bridge and a wing; the rotation of antenna; motor and helicopter rotor; the precession and nutation of ballistic missile[1-3]. Micro motion feature information which contained in radar echo of micro motion targets reflects the geometric structure and motion characteristics of the target, these information can be used in radar target feature extraction and target identification. The detection of micro motion feature is firstly realized by coherent laser radar, MicroDoppler laser radar has a series of advantages, such as far detection range; imaging available; the system structure is simple and portable[4-7]. Based on the above background, in this paper, the different Micro-Doppler mathematical models are established of four typical micro motion forms: vibration, rotation, tumble, cone[8-9], then, some simulations are carried out about the Micro-Doppler frequency of these four kinds of micro motion targets combined with an effective detection method in the field of laser radar: double pulse laser coherent detection, during the simulation, the MicroDoppler frequency features of different targets are compared and analyzed.

a Corresponding author : lishen9960@163.com

(C) 2016. The authors - Published by Atlantis Press 


\section{Micro-Doppler model of several typical micro motion forms}

\subsection{Vibrating target}

Vibration is a common micro motion form which can be seen in the vibration of the car engine, breathe and heartbeat of human body and so on.

The geometric graph of vibrating target and radar is showed on Figure 1. Radar is located on point $\mathrm{Q}, \mathrm{Q}$ is the origin of coordinate, the coordinate is $(\mathrm{A}, \mathrm{B}, \mathrm{C})$, the target coordinate is $(\mathrm{X}, \mathrm{Y}, \mathrm{Z})$, the origin is $\mathrm{O}$, the distance to $\mathrm{Q}$ is $\mathrm{R}_{0}, \alpha$ and $\beta$ are the azimuth and pitch angles of vibrating target coordinate relative to the radar coordinate system. The carrier frequency of radar is $f_{0}$, the velocity of electromagnetic wave is c, the amplitude of target is $D_{v}$, frequency is $f_{v}$, the azimuth and pitch angle of the vibration direction with respect to the point of $\mathrm{O}$ are $\alpha_{p}, \beta_{p}$.

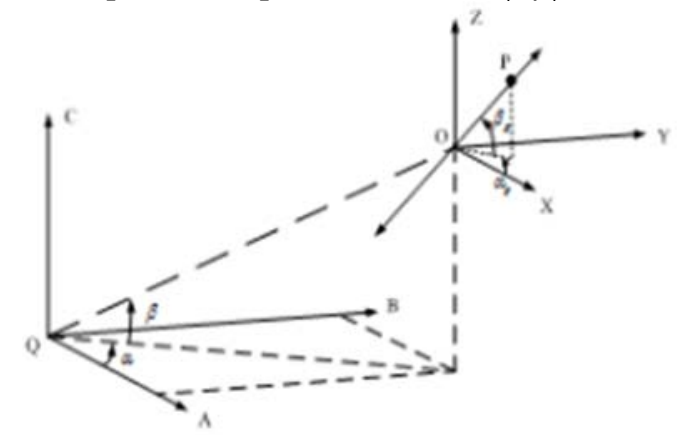

Figure 1. The geometric graph of vibrating target and radar.

The distance of radar to scattering point $\mathrm{P}$ is $R_{t}=R_{0}+D_{t}$, transform it to absolute value.

$$
R_{t}=\left|R_{t}\right|=\sqrt{\begin{array}{r}
\left(R_{0} \cos \alpha \cos \beta+D_{t} \cos \alpha_{p} \cos \beta_{p}\right)^{2}+\left(R_{0} \sin \alpha \cos \beta+D_{t} \sin \alpha_{p} \cos \beta_{p}\right)^{2} \\
+\left(R_{0} \sin \beta+D_{t} \sin \beta_{p}\right)^{2}
\end{array}}
$$

When $R_{0}>>D_{t}$, set up azimuth angle $\alpha=0$, pitch angle $\beta=0$, formula 1 becomes following form.

$$
R_{t}=\sqrt{\left(R_{0}^{2}+D_{t}^{2}+2 R_{0} D_{t} \cos \alpha_{p} \cos \beta\right)} \approx R_{0}+D_{t} \cos \alpha_{p} \cos \beta
$$

The vibration angular frequency of scattering point is $\omega_{v}=2 \pi f_{v}$, amplitude $D_{t}=D_{v} \sin \omega_{v} t$.

$$
R(t)=R_{t}=R_{0}+D_{v} \sin \omega_{v} t \cos \alpha_{p} \cos \beta
$$

Set $B=D_{v} \cos \alpha_{p} \cos \beta$, the echo formula of vibrating target can be derived.

$$
S(t)=\rho(x, y, z) \exp \left(j\left(2 \pi f_{0} t+4 \pi \cdot \frac{R(t)}{\lambda}\right)\right)=\rho(x, y, z) \exp \left(j \frac{4 \pi R_{0}}{\lambda}\right) \exp \left(j 2 \pi f_{0} t+B \sin \omega_{v} t\right)
$$

The velocity model of vibrating target is:

$$
v=2 \pi D_{v} f_{v} \cos \left(2 \pi f_{v} t\right) \cdot\left(\cos \alpha_{p} \cos \beta_{p}, \sin \alpha_{p} \cos \beta_{p}, \sin \beta_{p}\right)^{T}
$$

Based on equivalent scattering point model, simplify the micro motion target to a series of scatting points, a common formula of the target Micro-Doppler frequency can be derived.

$$
f_{\text {micro-doppler }}=\frac{2 f_{0}}{c}[\omega \times r]^{T} \cdot n
$$


In formula $6, f_{0}$ is the carrier frequency of radar. $c$ is the velocity of electromagnetic wave, $\omega=\left(\omega_{x}, \omega_{y}, \omega_{z}\right)^{\mathrm{T}}$ is rotating angular frequency of scattering point with respect to radar, $\mathrm{r}$ is the rotation distance. $n$ is the unit vector in the direction of the line of sight of radar, based on formula 6, the Micro-Doppler frequency of vibrating target can be derived.

$$
f_{\text {micro-doppler }}=\frac{2 f_{0}}{c}\left(v^{T} \cdot n\right)=\frac{4 \pi f_{0} f_{v} D_{v}}{c}\left(\cos \left(\alpha-\alpha_{p}\right) \cos \beta \cos \beta_{p}+\sin \beta \sin \beta_{p}\right) \cos \left(2 \pi f_{v} t\right)
$$

\subsection{Rotating target}

Rotation is also a typical micro motion form, which can be seen in the propeller of helicopter and the blade of a jet aircraft.

The geometric graph of rotating target and radar is showed on Figure 2. Radar is located on point $\mathrm{Q}, \mathrm{Q}$ is the origin of coordinate, the coordinate is $(\mathrm{A}, \mathrm{B}, \mathrm{C})$, the target coordinate is $(\mathrm{X}, \mathrm{Y}, \mathrm{Z})$, the origin is $\mathrm{O}$, the distance to is $\mathrm{Q}$ is $\mathrm{R}_{0}, \alpha$ and $\beta$ are the azimuth and pitch angles of rotating target coordinate relative to the radar coordinate system. The carrier frequency of radar is $f_{0}$, the velocity of electromagnetic wave is $c$.

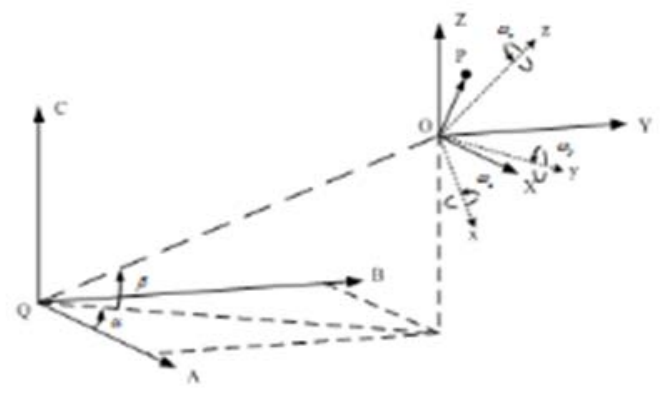

Figure 2. The geometric graph of rotating target and radar.

Define the rotation angular velocity of scattering point $\mathrm{P}$ on the target as $\omega=\left(\omega_{x}, \omega_{y}, \omega_{z}\right)$, $\mathrm{P}$ is located on $r_{0}=\left(x_{0}, y_{0}, z_{0}\right)$, then $\mathrm{P}$ rotate to a new position, which is $R_{I N I T} \cdot r_{0}$, the rotating unit angular velocity is:

$$
\omega^{\prime}=\left(\omega_{x}^{\prime}, \omega_{y}{ }^{\prime}, \omega_{z}{ }^{\prime}\right)=\frac{R_{I N I T} \cdot r_{0}}{\|\omega\|}
$$

In formula $8, \Omega=\|\omega\|$ is the modulus of angular velocity, $R_{I N I T}$ is the initial rotation matrix, which decided by Euler angle, as shown on Figure 3, define the rotation angular round $\mathrm{z}$ axis as $\phi$; the rotation angular round $\mathrm{x}$ axis as $\theta$; the rotation angular round $\mathrm{z}$ axis as $\psi$.
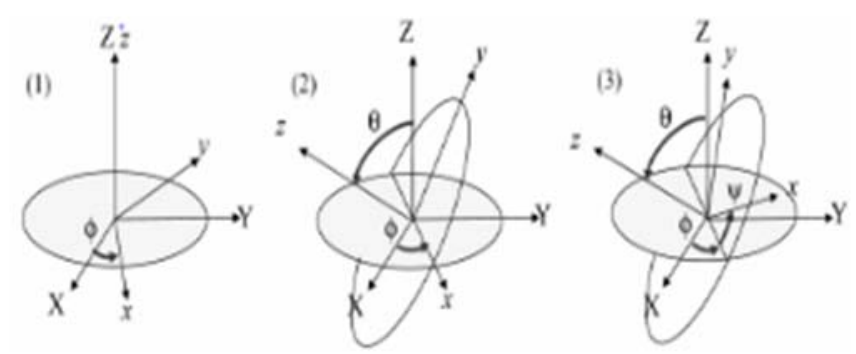

Figure 3. Euler angle.

The initial rotation matrix is: 


$$
R_{I N I T}=\left[\begin{array}{lll}
a_{11} & a_{12} & a_{13} \\
a_{21} & a_{22} & a_{23} \\
a_{31} & a_{32} & a_{33}
\end{array}\right]
$$

In formula 9:

$$
\left\{\begin{array}{c}
a_{11}=\cos \phi \cos \psi-\sin \phi \cos \theta \sin \psi \\
a_{12}=-\cos \phi \sin \psi-\sin \phi \cos \theta \cos \psi \\
a_{13}=\sin \phi \sin \theta \\
a_{21}=\sin \phi \cos \psi+\cos \phi \cos \theta \sin \psi \\
a_{22}=-\sin \phi \sin \psi+\cos \phi \cos \theta \cos \psi \\
a_{23}=-\cos \phi \sin \theta \\
a_{31}=\sin \theta \sin \psi \\
a_{32}=\sin \theta \cos \psi \\
a_{33}=\cos \theta
\end{array}\right\}
$$

Time $t$, the target rotate to a new position.

$$
r(t)=R_{t} \cdot R_{I N I T} \cdot r_{0}
$$

Define the rotation matrix as:

$$
R_{t}=I+\omega_{*}^{\prime} \sin \Omega t+\omega_{*}^{\prime 2}(1-\cos \Omega t)
$$

In formula $12, \omega_{*}^{\prime}$ is the skew symmetric matrix, which defined as:

$$
\omega_{*}^{\prime}=\left[\begin{array}{ccc}
0 & -\omega_{z}^{\prime} & \omega_{y}^{\prime} \\
\omega_{z}^{\prime} & 0 & -\omega_{x}^{\prime} \\
-\omega_{y}^{\prime} & \omega_{x}^{\prime} & 0
\end{array}\right]
$$

Based on formula 6, the Micro-Doppler frequency of rotating target can be derived.

$$
\begin{aligned}
f_{\text {micro-doppler }}= & \frac{2 f_{0}}{c} \cdot\left[\Omega \omega^{\prime} \times r\right]^{T} \cdot n=\frac{2 f_{0}}{c} \cdot\left(\Omega \omega_{*}^{\prime} \cdot r\right)^{T} \cdot n=\frac{2 f_{0}}{c} \cdot\left[\Omega \omega_{*}^{\prime} \cdot R_{t} \cdot R_{I N I T} \cdot r_{0}\right]^{T} \cdot n \\
& =\frac{2 f_{0} \Omega}{c} \cdot\left\{\left[\omega_{*}^{\prime 2} \sin \Omega t-\omega_{*}^{\prime 3} \cos \Omega t+\omega_{*}^{\prime}\left(I+\omega_{*}^{2}\right)\right] \cdot R_{I N I T} \cdot r_{0}\right\}^{T} \cdot n
\end{aligned}
$$

If the skew symmetric matrix is defined as a unit matrix, then $\omega_{*}^{3}=-\omega_{*}^{\prime}$, formula 14 can be simplified into formula 15.

$$
f_{\text {micro-doppler }}=\frac{2 f_{0}}{c} \cdot \Omega \omega_{*}^{\prime}\left(\omega_{*}^{\prime} \sin \Omega t+I \cos \Omega t\right) \cdot R_{I N I T} \cdot r_{0} \cdot(\cos \alpha \cos \beta, \sin \alpha \cos \beta, \sin \beta)^{T}
$$

\subsection{Tumbling target}

Tumble can be seen as a special form of rotation, it can be simplified as a rotating target attached to a translational and accelerated motion, such as a moving spacecraft in space. 


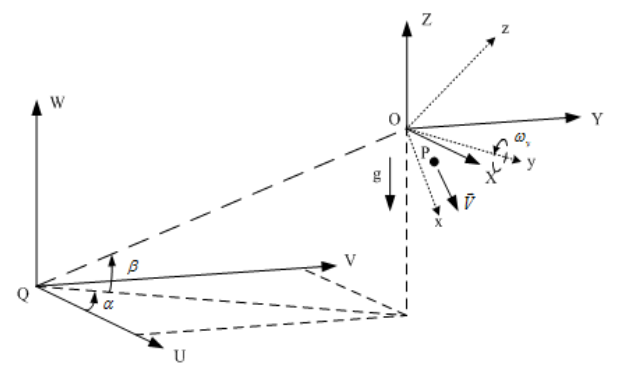

Figure 4. The geometric graph of tumbling target and radar.

The geometric graph of tumbling target and radar is showed on Figure 4. Radar is located on point $\mathrm{Q}, \mathrm{Q}$ is the origin of coordinate, the coordinate is $(\mathrm{U}, \mathrm{V}, \mathrm{W})$, the target coordinate is $(\mathrm{X}, \mathrm{Y}, \mathrm{Z})$, the origin is $\mathrm{O}$, the distance to is $\mathrm{Q}$ is $\mathrm{R}_{0}, \alpha$ and $\beta$ are the azimuth and pitch angles of tumbling target coordinate relative to the radar coordinate system. The carrier frequency of radar is $f_{0}$, the velocity of electromagnetic wave is $c$. Define the target has a rotation round $y$ axis, a translation along $\mathrm{x}$ axis, it has a vertical downward acceleration $g$ because of gravity, define the initial position of scattering point $\mathrm{P}$ on the target is $r_{0}=[x, y, z]^{\mathrm{T}}$, the initial velocity is $v_{*}=v \cdot\left[\alpha_{11}, \alpha_{21}, a_{31}\right]^{\mathrm{T}}, \alpha_{11}, \alpha_{21}, a_{31}$ are defined by formula 10. $\omega=\omega_{y}, \omega=\omega \cdot\left[\alpha_{11}, \alpha_{22}, \alpha_{33}\right]^{\mathrm{T}}$, time $t$, point $\mathrm{P}$ moved to a new position.

$$
r(t)=R_{I N I T}\left[\begin{array}{ccc}
\cos \omega t & 0 & \sin \omega t \\
0 & 1 & 0 \\
-\sin \omega t & 0 & \cos \omega t
\end{array}\right] r_{0}
$$

at this time, the velocity of point $\mathrm{P}$ changed into $v_{*}=\left(v a_{11}, v a_{21}, v a_{31}-g t\right)^{\mathrm{T}}$, the Doppler frequency of tumbling target can be derived.

$$
f_{D}=\frac{2 f_{0}}{c}\left[v_{*}+\omega^{\prime} \times r\right]^{T}=\frac{2 f_{0}}{c}\left(-g t \sin \beta+t_{1}\right)+\frac{2 f_{0} \omega}{c}\left[\left(x t_{2}+z t_{3}\right) \cos \omega t+\left(z t_{2}-x t_{3}\right) \sin \omega t\right](17
$$

Three time variables in formula 17 are defined as:

$$
\left\{\begin{array}{c}
t_{1}=v\left[\left(a_{11} \cos \alpha+a_{21} \sin \alpha\right) \cos \beta+a_{31} \sin \beta\right] \\
t_{2}=\left[\left(a_{22} a_{31}-a_{32} a_{21} \cos \alpha\right)+\left(a_{32} a_{11}-a_{12} a_{31}\right) \sin \alpha\right] \cos \beta+\left(a_{12} a_{21}-a_{22} a_{11}\right) \sin \beta \\
t_{3}=\left[\left(a_{22} a_{33}-a_{32} a_{23} \cos \alpha\right)+\left(a_{32} a_{13}-a_{12} a_{33}\right) \sin \alpha\right] \cos \beta+\left(a_{12} a_{23}-a_{22} a_{13}\right) \sin \beta
\end{array}\right\}
$$

The velocity model of the tumbling target is:

$$
v(t)=-g t \cdot \sin \beta+t_{1}+\omega\left[\left(x t_{2}+z t_{3}\right) \cos \omega t+\left(z t_{2}-x t_{3}\right) \sin \omega t\right]
$$

Based on the velocity model, the Micro-Doppler frequency of tumbling target can be derived.

$$
f_{\text {micro-doppler }}=-\frac{2 f_{0}}{c} g t \cdot \sin \beta+\frac{2 f_{0}}{c} \omega\left[\left(x t_{2}+z t_{3}\right) \cos \omega t+\left(z t_{2}-x t_{3}\right) \sin \omega t\right]
$$

\subsection{Coning target}

Cone can also be seen as a special form of rotation, it is a kind of movement that one point of the target is fixed, the other parts rotate round a certain axis, such as the movement of the blender; the movement of tank gun barrel after the bullet is fired out. 


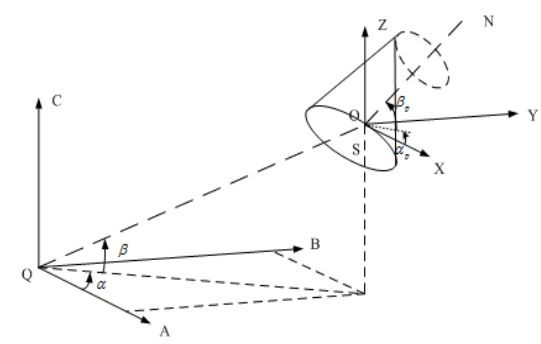

Figure 5. The geometric graph of coning target and radar.

The geometric graph of coning target and radar is showed on Figure 5. Radar is located on point Q, $\mathrm{Q}$ is the origin of coordinate, the coordinate is $(\mathrm{U}, \mathrm{V}, \mathrm{W})$, the target coordinate is $(\mathrm{X}, \mathrm{Y}, \mathrm{Z})$, the origin is $\mathrm{O}$, the distance to is $\mathrm{Q}$ is $\mathrm{R}_{0}, \alpha$ and $\beta$ are the azimuth and pitch angles of coning target coordinate relative to the radar coordinate system. The carrier frequency of radar is $f_{0}$, the velocity of electromagnetic wave is $c$. Point $\mathrm{P}$ on the target rotates round axis $\mathrm{SN}, \mathrm{S}$ is fixed, the azimuth angle of SN with respect to the coordinate axis is $\alpha_{N}$, the pitch angle is $\beta_{N}$, the unit vector along the rotation axis can be defined as:

$$
e=\left(\cos \beta_{N} \cos \alpha_{N}, \cos \beta_{N} \sin \alpha_{N}, \sin \beta_{N}\right)
$$

Define the initial position of point $\mathrm{P}$ as $r_{0}=[x, y, z]^{\mathrm{T}}$, the coordinate of $\mathrm{S}$ is $\left(\mathrm{x}=0, \mathrm{y}=0, \mathrm{z}=\mathrm{z}_{0}\right)$, $\omega_{c}=2 \pi f_{c}$ is the coning angular velocity, at time $\mathrm{t}$, point $\mathrm{P}$ moves to a new position.

$$
r(t)=R_{c} \cdot R_{I N I T} \cdot\left[x, y, z-z_{0}\right]
$$

In formula 22, define $R_{c}$ as the cone matrix.

$$
R_{c}=I+e^{\wedge} \sin \omega_{c} t+e^{\wedge^{2}}\left(1-\cos \omega_{c} t\right)
$$

Define $e^{\wedge}$ as the skew symmetric matrix.

$$
e^{\wedge}=\left[\begin{array}{ccc}
0 & -\sin \beta_{N} & \sin \alpha_{N} \cos \beta_{N} \\
\sin \beta_{N} & 0 & -\cos \alpha_{N} \cos \beta_{N} \\
-\sin \alpha_{N} \cos \beta_{N} & \cos \alpha_{N} \cos \beta_{N} & 0
\end{array}\right]
$$

The Micro-Doppler frequency of coning target can be derived.

$$
\begin{aligned}
f_{\text {micro-doppler }} & =\frac{2 f_{0}}{c}\left[\frac{d}{d t} r(t)\right]^{T} \cdot n=\frac{2 f_{0}}{c}\left\{\left[\frac{d}{d t} R_{t}\right] R_{I N I T} \cdot\left[x, y, z-z_{0}\right]^{T}\right\}^{T} \cdot n \\
& =\frac{2 f_{0} \omega_{c}}{c}\left\{\left(e^{\wedge} \cos \omega t+e^{\wedge^{2}} \sin \omega t\right) R_{I N I T} \cdot\left[x, y, z-z_{0}\right]^{T}\right\}^{T}
\end{aligned}
$$

\section{Micro-Doppler signal simulation of point targets}

Through an effective method to detect the Micro-Doppler feature of the target is the basis for simulation research. Based on the traditional time-frequency analysis algorithm, a new detection scheme is introduced in this paper: double pulse laser coherent detection[10]. The basic idea of this algorithm can be described as: concerning the micro motion target as the point target, laser emits double pulse laser signals constantly to probe the target, by solving the phase difference between the probe signal and the local oscillator reference signal, the micro motion velocity and the Micro- 
Doppler frequency of the target is obtained. According to the detecting principle of this algorithm, the signal phase differencecan $\Delta \varphi$ be derived:

$$
\Delta \varphi=\operatorname{angle}\left[\exp \left(-j \frac{4 \pi}{\lambda} \Delta T v\left(\frac{p}{f_{P R F}}\right)\right) \cdot \exp \left(j \frac{4 \pi}{\lambda} \Delta T v\left(\frac{p}{f_{P R F}}\right)\right)\right]
$$

In formula 26, $v$ is defined as the estimation of instantaneous velocity of micro motion target at the time when double pulse group $\mathrm{P}$ is emitted, then, the Micro-Doppler frequency of target which derived by phase difference is:

$$
f d=\frac{\Delta \varphi}{2 \pi \cdot \Delta T}
$$

In this section, combine the principle of double pulse laser coherent detection and the mathematical model which derived in section 2, the Micro-Doppler time frequency spectrum graphs of four kinds of micro motion targets are obtained by simulation. During the simulation, by changing the values of different input parameters in the micro motion model, the influence of these parameters on the Micro-Doppler frequency is studied, the features of Micro-Doppler time frequency spectrum of different targets are compared and analyzed.

\subsection{Vibrating target}

Set the basic parameters of the detection system: laser wavelength $\lambda=1.064 \mathrm{um}$; double pulse repetition frequency $f_{P R F}=1000 \mathrm{~Hz}$; double pulse interval $\triangle T=50 \mathrm{us}$; number of pulses $P=2000$.

\subsubsection{Influence of vibration angular frequency}

During simulation, set the amplitude to a constant value $A m=0.02 \mathrm{~m}$; set the angular frequency to $\omega_{1}=2 \pi \mathrm{rad} / \mathrm{s}, \omega_{2}=4 \pi \mathrm{rad} / \mathrm{s}$; simulate and obtain the Micro-Doppler frequency separately.

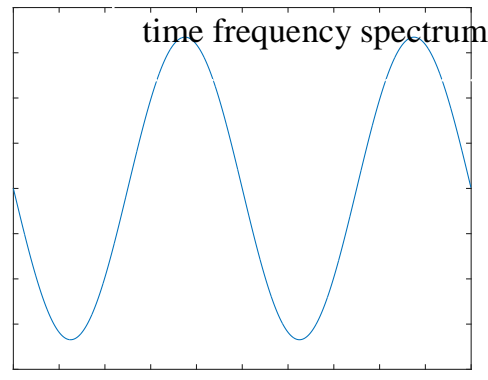

$\mathrm{t} / \mathrm{s}$

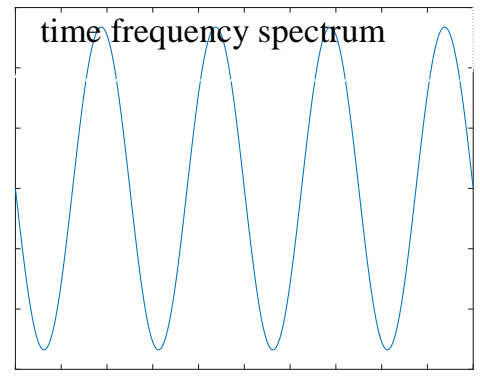

$\mathrm{t} / \mathrm{s}$

Figure 6. $\omega_{1}=2 \pi \mathrm{rad} / \mathrm{s}$.

Figure 7. $\omega_{2}=4 \pi \mathrm{rad} / \mathrm{s}$.

Compare Figure 6 with Figure 7, when amplitude is invariant, with the increase of the vibration angular frequency, the maximum Micro-Doppler frequency increased. Compared with Figure 6, in Figure 7, the reduction of the spectrum period can also reflect the improvement of angular frequency.

\subsubsection{Influence of amplitude}

During simulation, set the angular frequency to a constant value $\omega=4 \pi \mathrm{rad} / \mathrm{s}$; set the amplitude to $A m_{1}=0.01 \mathrm{~m}, A m_{2}=0.03 \mathrm{~m}$; simulate and obtain the Micro-Doppler frequency separately. 


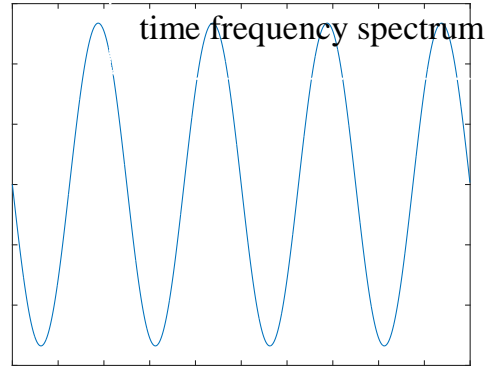

$\mathrm{t} / \mathrm{s}$

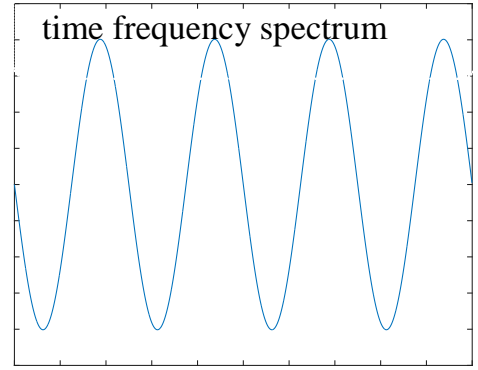

$\mathrm{t} / \mathrm{s}$

Figure 8. $A m_{1}=0.01 \mathrm{~m}$.

Figure 9. $A m_{2}=0.03 \mathrm{~m}$.

Compare Figure 8 with Figure 9, when the angular frequency is constant, change the amplitude of the target, the maximum Micro-Doppler frequency increased with the increase of the amplitude.

\subsection{Rotating target}

Set the basic parameters of the detection system: laser wavelength $\lambda=1.064 \mathrm{um}$; double pulse repetition frequency $f_{P R F}=1000 \mathrm{~Hz}$; double pulse interval $\Delta T=50 \mathrm{us}$; number of pulses $P=2000$, Euler angle: $\left(\phi=30^{\circ}, \theta=20^{\circ}, \psi=20^{\circ}\right)$.

During simulation, three scattering points on the rotating target are selected. When graphing, use a thin full line to represent the time frequency spectrum line of the point at $(0,0,0)$; use a dotted line to represent the time frequency spectrum line of the point at $(0.8,0.4,0.6)$; use a thick full line to represent the time frequency spectrum line of the point at $(-0.8,-0.4,-0.6)$. The Micro-Doppler frequency of the rotating target is mainly related to the rotation angular velocity, set the basic parameters to constant values; set the angular velocity to $\omega_{1}=(\pi, \pi, \pi), \omega_{2}=(\pi, 2 \pi, 2 \pi)$; simulate and obtain the Micro-Doppler frequency separately.

Compare Figure 10 with Figure 11, the change of the rotation angular velocity will lead to the change of the Micro-Doppler frequency of the rotating target, the maximum Micro-Doppler frequency increased with the increase of the angular velocity. At the same time, the period of the time frequency spectrum is different in different angular velocity, period of rotation: $\mathrm{T}_{1}=\frac{2 \pi}{\omega_{1}}=1.154 \mathrm{~s} ; \mathrm{T}_{2}=\frac{2 \pi}{\omega_{2}}=0.667 \mathrm{~s}$, which consistent with the simulation result in Figure 10 and Figure 11. The initial phase and amplitude of the time frequency spectrum of the scattering points with different coordinates are also different, the point on the rotation axis with the coordinate $(0,0,0)$, its time frequency spectrum is a straight line on the zero frequency.

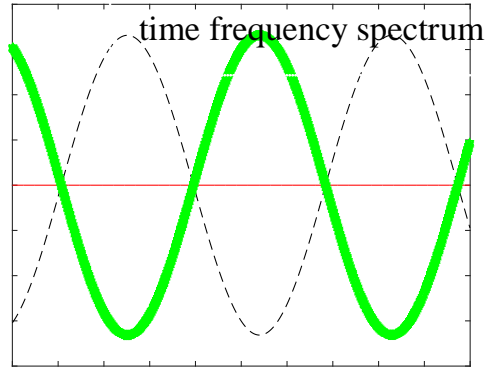

$\mathrm{t} / \mathrm{s}$

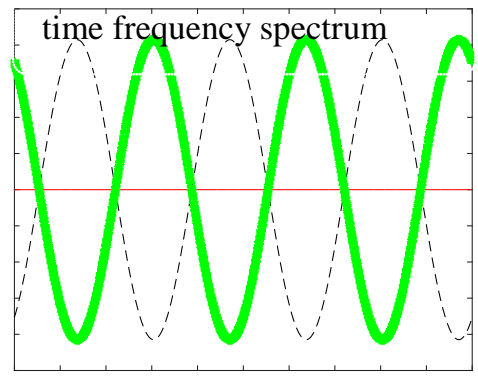

$\mathrm{t} / \mathrm{s}$

Figure 10. $\omega_{1}=(\pi, \pi, \pi)$.

Figure 11. $\omega_{2}=(\pi, 2 \pi, 2 \pi)$. 


\subsection{Tumbling target}

Set the basic parameters of the detection system: laser wavelength $\lambda=1.064 \mathrm{um}$; double pulse repetition frequency $f_{P R F}=1000 \mathrm{~Hz}$; double pulse interval $\Delta T=50$ us; number of pulses $P=2000$, Euler angle: $\left(\phi=30^{\circ}, \theta=20^{\circ}, \psi=20^{\circ}\right)$.

During simulation, three scattering points on the tumbling target are selected. When graphing, use a thin full line to represent the time frequency spectrum line of the point at $(0,0,0)$; use a dotted line to represent the time frequency spectrum line of the point at $(0.4,0.2,0.3)$; use a thick full line to represent the time frequency spectrum line of the point at $(-0.4,-0.2,-0.3)$.

\subsubsection{Influence of tumble angular velocity}

During simulation,set the acceleration to a constant value $g=9.8 \mathrm{~m} / \mathrm{s}^{2}$; set the angular velocity to $\omega_{y 1}=2 \pi \mathrm{rad} / \mathrm{s} ; \omega_{y 2}=4 \pi \mathrm{rad} / \mathrm{s} ;$ simulate and obtain the Micro-Doppler frequency separately.

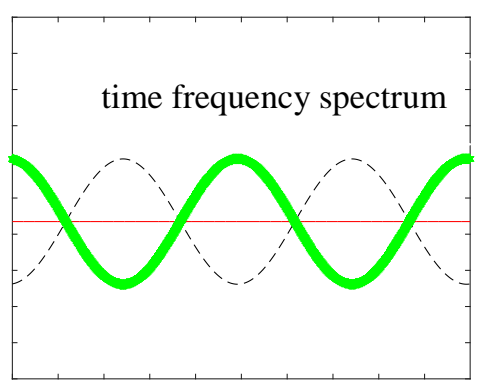

$\mathrm{t} / \mathrm{s}$

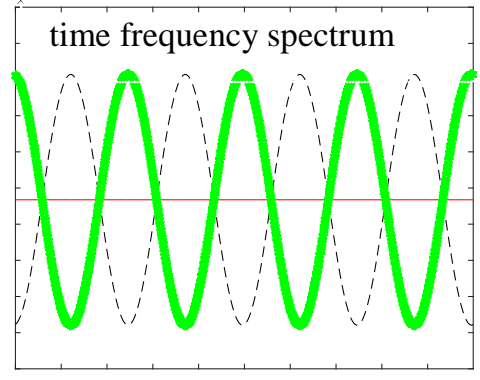

$\mathrm{t} / \mathrm{s}$

Figure 12. $\omega_{y 1}=2 \pi \mathrm{rad} / \mathrm{s}$.

Figure 13. $\omega_{y 2}=4 \pi \mathrm{rad} / \mathrm{s}$.

Compare Figure 12 with Figure 13, only change the tumble angular velocity of scattering point, the maximum Micro-Doppler frequency increased with the increase of the angular velocity and the period of the time frequency spectrum reduced, period of tumble: $\mathrm{T}_{1}=\frac{2 \pi}{\omega_{y_{1}}}=1 \mathrm{~s} ; \mathrm{T}_{2}=\frac{2 \pi}{\omega_{y_{2}}}=0.5 \mathrm{~s}$; which consistent with the simulation result in Figure 12 and Figure 13. For the point on the tumble axis with the coordinate $(0,0,0)$, its time frequency spectrum is no longer a straight line on the zero frequency, but a straight line with a certain frequency deviation.

\subsubsection{Influence of acceleration}

During simulation, set the angular velocity to a constant value $\omega_{y}=4 \pi \mathrm{rad} / \mathrm{s}$; set the acceleration to $g_{1}=19.6 \mathrm{~m} / \mathrm{s}^{2}, g_{2}=39.2 \mathrm{~m} / \mathrm{s}^{2}$, simulate and obtain the Micro-Doppler frequency separately.

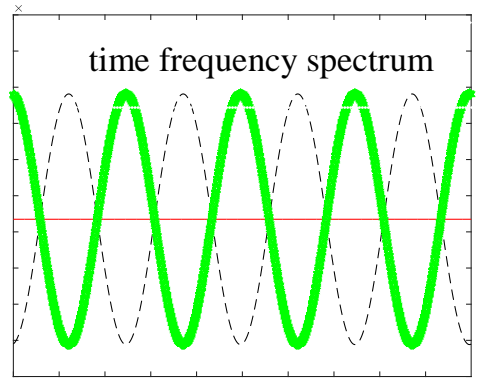

$\mathrm{t} / \mathrm{s}$

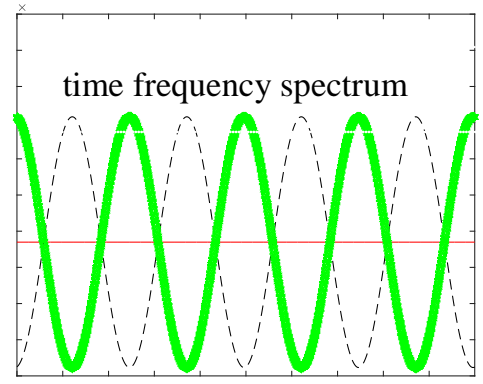

$\mathrm{t} / \mathrm{s}$

Figure 14. $g_{1}=19.6 \mathrm{~m} / \mathrm{s}^{2}$.

Figure 15. $g_{2}=39.2 \mathrm{~m} / \mathrm{s}^{2}$. 
Compare Figure 14 with Figure 15, For the point on the tumble axis with the coordinate $(0,0,0)$, its time frequency spectrum is a straight line with a certain frequency deviation, the frequency deviation increased with the increase of acceleration, this Micro-Doppler feature can be used as a typical feature to distinguish the rotating target and the tumbling target.

\subsection{Coning target}

Set the basic parameters of the detection system: laser wavelength $\lambda=1.064 \mathrm{um}$; double pulse repetition frequency $f_{P R F}=1000 \mathrm{~Hz}$; double pulse interval $\triangle T=50 \mathrm{us}$; number of pulses $P=2000$, Euler angle: $\left(\phi=30^{\circ}, \theta=20^{\circ}, \psi=20^{\circ}\right)$.

During simulation, three scattering points on the tumbling target are selected. When graphing, use a thin full line to represent the time frequency spectrum line of the point at $(0,0,0)$; use a dotted line to represent the time frequency spectrum line of the point at $(0.4,0.2,0.3)$; use a thick full line to represent the time frequency spectrum line of the point at $(-0.4,-0.2,-0.3)$.

During simulation, three scattering points on the coning target are selected. When graphing, use a thin full line to represent the time frequency spectrum line of the point at $(0,0,0)$; use a dotted line to represent the time frequency spectrum line of the point at $(-0.5,0.3,0.4)$; use a thick full line to represent the time frequency spectrum line of the point at $(0.5,-0.3,-0.4)$. The Micro-Doppler frequency of the coning target is mainly related to the cone angular velocity, set the basic parameters to constant values; set the angular velocity to $\omega_{1}=4 \pi \mathrm{rad} / \mathrm{s} ; \omega_{2}=6 \pi \mathrm{rad} / \mathrm{s}$; simulate and obtain the MicroDoppler frequency separately.

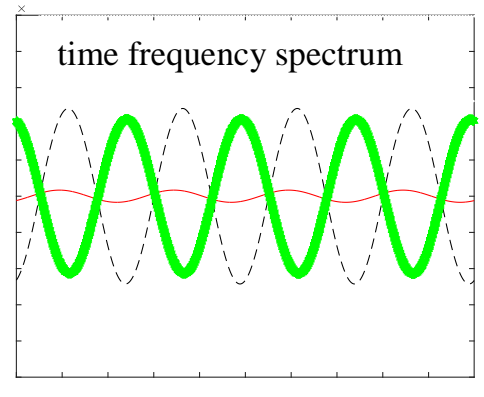

$\mathrm{t} / \mathrm{s}$

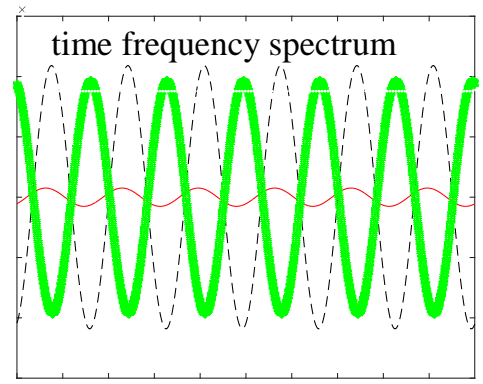

$\mathrm{t} / \mathrm{s}$

Figure 16. $\omega_{1}=4 \pi \mathrm{rad} / \mathrm{s}$.

Figure 17. $\omega_{2}=6 \pi \mathrm{rad} / \mathrm{s}$.

Compare Figure 16 with Figure 17, the maximum Micro-Doppler frequency increased with the increase of the cone angular velocity and the period of the time frequency spectrum reduced, period of cone: $\mathrm{T}_{1}=\frac{2 \pi}{\omega_{1}}=0.5 \mathrm{~s} ; \mathrm{T}_{1}=\frac{2 \pi}{\omega_{2}}=0.33 \mathrm{~s}$; which consistent with the simulation result in Figure 16 and Figure 17. On the other hand, two scattering points on the symmetry of the origin which coordinates are ($0.5,0.3,0.4)$ and $(0.5,-0.3,-0.4)$ have an opposite instantaneous frequency, otherwise, their corresponding maximum Micro-Doppler frequency absolute value are no longer the same. For the point on the cone axis with the coordinate $(0,0,0)$, its time frequency spectrum is a kind of period curve which has a small fluctuation round zero frequency, no longer a straight line on the zero frequency. This two Micro-Doppler frequency features can be used as a typical feature to distinguish coning target from other micro motion targets.

\section{Conclusions}

This paper used a method of combining theoretical analysis and simulation research. First, a MicroDoppler mathematical model is established of the point target and radar in four kinds of micro motion forms of vibration, rotation, tumble and cone. A new algorithm for detecting the Micro-Doppler 
feature of the target is introduced which called double pulse laser coherent detection. By using the principle of calculating the Micro-Doppler frequency in this algorithm, the echo signals of the four kinds of micro motion targets are simulated respectively. From the simulation result it can be seen that different input parameters can influence the Micro-Doppler frequency of the target, on the other hand , the Micro-Doppler time frequency spectrum of four different kinds of micro motion targets are different, which reflected that each kind of target has its own Micro-Doppler feature, The classification and identification of different targets may be realized by using these Micro-Doppler features.

\section{References}

1. Xing-yong CHEN. Research on Radar Signature Extraction from Target with Micro-motions [D]. National University of Defence Technology (2004).

2. Meng WEI. Micro-Doppler Effect Analysis and Parameter Extraction [D]. University of Electronic Science and Technology of China (2010).

3. Ting FU. Research on Classification of Target based on Micro-Doppler [D]. Xidian University (2011).

4. V.V.Molebny, G.W.Kamerman, O.Steinvall. Laser remote sensing: yesterday, today and tomorrow [J].Electronics and Nanotechnology, April 68-73 (2011).

5. R.M.Marino, W.R.Davis. Jigsaw: A Foliage-Penetrating 3D imaging laser radar system [J]. Lincoln Laboratory Journal, 5(1) 23-36(2005).

6. Peng-fei ZHANG, Jing-yan PAN, Tao ZHANG. Study on the object detection with the coherent lidar system [J]. LASER \& INFRARED, 45(7) 753-756(2015).

7. Jing DONG, Rui CHEN, Xiao-long LI. Lidar Coherent Detection and Feature Extraction of Moving Target Based on Micro-Doppler Effect [J]. Chinese Journal of Lasers, 39(10)1014001-11014001-4(2015).

8. V.C.Chen, F.Y.LI, S.S.HO. Micro-Doppler effect in radar: phenomenon, model and simulation study [J].IEEE transactions on Aerospace and Electronic Systems, 42(1)2-21(2006).

9. V.C. Chen. Micro-Doppler effect of micro-motion dynamics: a review[C]. Proceedings of SPIE, 5102 240-249(2003).

10. N.S.Prasad, V.Rudd, S.Shald. Doublet pulse coherent laser radar for tracking of resident space objects[C].Proceedings of the Advanced Maui Optical, space Surveillance Technologies Conference (2014). 